\title{
Narrow escape problem for Brownian particles in a microsphere with internal circulation
}

\author{
C. A. Klettner \\ Department of Mechanical Engineering, University College London, Torrington Place, London, WC1E 7JE, United Kingdom
}

(Received 8 May 2019; published 14 October 2019)

\begin{abstract}
This paper considers the narrow escape problem of a Brownian particle within a two-dimensional domain with two escape windows and an internal circulation modeled by the flow within a Hill's vortex. To account for the spatially inhomogeneous flow within the domain, a Lagrangian study is undertaken using the complete equations of motion for a dense particle which is necessary to distinguish between the various regimes as the strength of the internal circulation is varied. For very low internal circulation the particle undergoes the conventional narrow escape problem, and agreement is good with the asymptotic expression. As the internal circulation is increased, regimes are identified with different scaling for the mean escape time. The potential application of this for drug delivery (were nanoparticles are encased in a microsphere) is discussed.
\end{abstract}

DOI: 10.1103/PhysRevE.100.043107

\section{INTRODUCTION}

The narrow escape problem, which can be dated back to Rayleigh [1], involves a particle undergoing Brownian motion in an enclosed domain, where the boundaries are all reflecting except a small window, through which the particle can escape [2]. There are many biological applications where this occurs including calcium decay in dendritic spines [2] and phototransduction [3], resulting in an intense period of research in the field in the last decade. There are also many variations of the problem which can be studied, including having multiple windows [4], imperfect trapping [5], and mixed transport boundaries [6]. Due to the very small time steps required for this problem (to resolve the Brownian motion timescales) the narrow escape problem is usually approached using asymptotic methods (see review in Ref. [7]), although some Monte Carlo studies have also been made [8]. Only recently have studies been undertaken to look at the effect of having a drift in the domain, which has included planar flow [9] and flow in an annulus [10].

Particles moving within and around spherical bubbles and vortices have been studied in the context of industrial and environmental processes. Small water droplets dispersing and depositing in a rising bubble, where the internal circulation was modeled using a Hill's vortex, were studied using an Eulerian transport model [11] and a Lagrangian model [12]. It was found that the Eulerian model overpredicted the time taken for particles to be deposited on the bubble. The dispersion of sedimenting small dense particles through and around a rising Hill's vortex was also studied [13]. Related to this work is the enhanced diffusivity of impurities in RayleighBenard convection cells [14].

In this work we consider the narrow escape problem of a Brownian particle within a moving microsphere that has internal circulation modeled as a Hill's vortex. To account for this spatially inhomogeneous flow, the full equations of motion for the particle are required, and a detailed study is made when the various force contributions are important as the strength of the internal circulation is varied and regimes are identified with different scaling for the mean escape time. The potential application of this study to drug delivery is then discussed.

This paper is structured as follows: The numerical modeling is described in Sec. II, which will include the model for the fluid phase, the equations of motion for a dense particle and details of the numerical simulations. In Sec. III the results are presented. Discussion and conclusions are given in Sec. IV.

\section{METHODOLOGY}

In this section the modeling of the fluid phase and the equations of motion for the nanoparticle are described. After this, details of the numerical simulations are given.

\section{A. Fluid phase}

In this work the fluid phase will be modeled as the flow within a Hill's vortex [15]. The Stokes stream function $\boldsymbol{\Psi}$ for a Hill's vortex with radius $R$, propagating with speed $U$ in the $x$ direction [see Fig. 1(a) for the schematic] is [13]

$$
\boldsymbol{\Psi}(x, y)=-\frac{3 U}{4 R^{2}}\left(y^{4}+y^{2} x^{2}-\frac{5}{3} y^{2} R^{2}\right) .
$$

In Fig. 1(b) the streamlines of the internal circulation are plotted. In the frame of reference of the moving sphere, the velocities in a cylindrical coordinate system in the sphere are

$$
u_{x}=-\frac{3 U}{4}\left[4\left(\frac{y}{R}\right)^{2}+2\left(\frac{x}{R}\right)^{2}-\frac{10}{3}\right]-U
$$

and

$$
u_{y}=-\frac{3 U}{2 R^{2}} x y .
$$

The azimuthal component of vorticity is

$$
\omega_{\theta}=\frac{15 U y}{2 R^{2}} .
$$




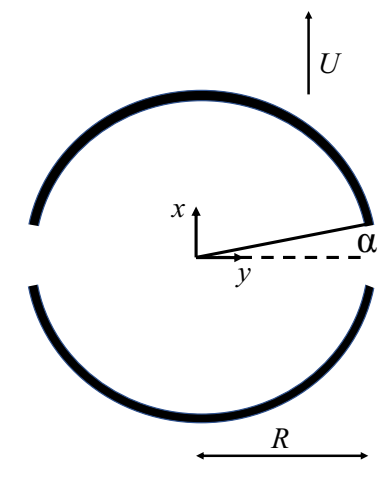

(a)

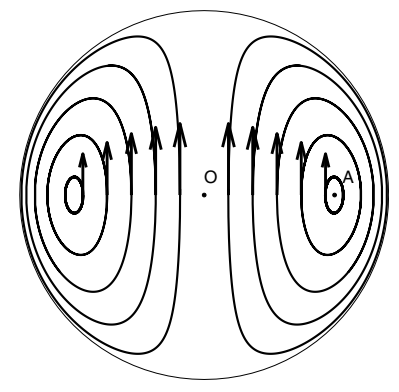

(b)
FIG. 1. (a) Schematic of the narrow escape problem of a Brownian particle within a microsphere (of radius $R$, traveling at speed $U$ ) of fluid. The Brownian particle moves according to (14) and is reflected by all boundaries except two windows, of angle $2 \alpha$, on either side through which it can escape. (b) Streamlines of a Hill's vortex showing the circulatory pattern within a microsphere due to its movement through the ambient fluid.

As can be seen from (2)-(4), $U$ is not only the speed of the microsphere but also a measure of the strength of the internal circulation of the fluid.

\section{B. Particle modeling}

The position of a particle (with mass $m_{p}$ ) with radius $r_{p}$ and density $\rho_{p}$ moving in a fluid with density $\rho_{f}$ and dynamic viscosity $\mu_{f}$ is given by

$$
\frac{d x}{d t}=\boldsymbol{v}
$$

and

$$
m_{p} \frac{d \boldsymbol{v}}{d t}=\boldsymbol{F}_{D}+\boldsymbol{F}_{B}+\boldsymbol{F}_{A}+\boldsymbol{F}_{I}+\boldsymbol{F}_{S}+\boldsymbol{F}_{H}+\boldsymbol{F}_{g},
$$

where $\boldsymbol{F}_{D}, \boldsymbol{F}_{B}, \boldsymbol{F}_{A}, \boldsymbol{F}_{I}, \boldsymbol{F}_{S}, \boldsymbol{F}_{H}$, and $\boldsymbol{F}_{g}$ are the drag, Brownian, added mass, inertial, shear induced lift, history, and gravitational force, respectively. This is the same equation as in Ref. [13], except that this formulation also includes the Brownian motion force. History effects are neglected, which is appropriate when $9 \rho_{f} / 2 \rho_{p} \ll 1$, as is the case in this study [16]. The particle is assumed to stay in the horizontal, twodimensional plane shown in Fig. 1(b), so gravitational effects are not considered. The drag force is given by the Stokes drag law, which is

$$
\boldsymbol{F}_{D}=\frac{6 \mu_{f} \pi r_{p}}{C_{c}}(\boldsymbol{u}-\boldsymbol{v})
$$

where $\boldsymbol{u}$ and $\boldsymbol{v}$ are the fluid and particle velocity, respectively [17]. $C_{c}$ is the Cunningham correction factor, which is related to the mean free path, $\lambda$, as

$$
C_{c}=1+\frac{\lambda}{r_{p}}\left(1.257+0.4 e^{-1.1 r_{p} / \lambda}\right)
$$

and is significant for gases. The amplitudes of the Brownian motion force components are given in Ref. [18] as

$$
\boldsymbol{F}_{B}=\zeta \sqrt{\frac{2 k_{b}^{2} T_{f}^{2}}{D \Delta t}},
$$

where $\zeta$ is a zero-mean, unit-variance-independent Gaussian random number, and $\Delta t$ is the integration time step, where $T_{f}$ is the absolute temperature of the fluid, $k_{b}$ is the Boltzmann constant, and the diffusion coefficient is given by

$$
D=\frac{k_{b} T_{f} C_{c}}{6 \pi \mu_{f} r_{p}} .
$$

The added mass force is

$$
\boldsymbol{F}_{A}=\rho_{f} C_{m} \frac{4}{3} \pi r_{p}^{3}\left(\frac{D \boldsymbol{u}}{D t}-\frac{d \boldsymbol{v}}{d t}\right)
$$

where the added mass of the particle is important when $\rho_{p} / \rho_{f} \approx 1$. The added mass coefficient is $C_{m}=1 / 2$ for a spherical particle [13]. The shear-induced lift force is

$$
\boldsymbol{F}_{S}=\rho_{f} C_{L} \frac{4}{3} \pi r_{p}^{3}(\boldsymbol{u}-\boldsymbol{v}) \times \boldsymbol{\omega},
$$

where $C_{L}=2$ for a spherical particle [19]. The inertial term is

$$
\boldsymbol{F}_{I}=\frac{4}{3} \pi \rho_{f} r_{p}^{3} \frac{D \boldsymbol{u}}{D t} .
$$

The expressions for $\boldsymbol{F}_{A}$ and $\boldsymbol{F}_{I}$ can be simplified as the flow is steady in the frame of reference of the moving microsphere. The full particle equation of motion is therefore

$$
\begin{aligned}
\left(m_{p}\right. & \left.+m_{a}\right) \frac{d \boldsymbol{v}}{d t} \\
= & \underbrace{\frac{6 \mu_{f} \pi r_{p}}{C_{c}}(\boldsymbol{u}-\boldsymbol{v})}_{\boldsymbol{F}_{D}}+\underbrace{\zeta \sqrt{\frac{2 k_{b}^{2} T^{2}}{D \Delta t}}}_{\boldsymbol{F}_{B}} \\
& +\underbrace{\left(1+C_{m}\right) \frac{4}{3} \pi \rho_{f} r_{p}^{3} \boldsymbol{u} \cdot \nabla \boldsymbol{u}}_{\boldsymbol{F}_{I+A}}+\underbrace{\rho_{f} C_{L} \frac{4}{3} \pi r_{p}^{3}(\boldsymbol{u}-\boldsymbol{v}) \times \boldsymbol{\omega}}_{\boldsymbol{F}_{S}},
\end{aligned}
$$

where $m_{a}=4 / 3 C_{m} \pi r_{p}^{3}$. The equations of motion can be nondimensionalized using $\tilde{\boldsymbol{v}}=\boldsymbol{v} / U, \tilde{\boldsymbol{u}}=\boldsymbol{u} / U, \tilde{\boldsymbol{x}}=\boldsymbol{x} / R$, and $\tau=t D / R^{2}$, which reduces (14) to

$$
\begin{aligned}
\frac{d \tilde{\boldsymbol{v}}}{d \tau}= & \underbrace{\left(\frac{\tau_{R}}{\tau_{B} C_{c}}\right)(\tilde{\boldsymbol{u}}-\tilde{\boldsymbol{v}})}_{\boldsymbol{F}_{D}}+\underbrace{\left(\frac{|\boldsymbol{v}|^{\prime}}{U}\right)\left(\frac{\tau_{R}}{\tau_{B}}\right) \zeta \sqrt{\frac{4}{3 C_{c}}}}_{\boldsymbol{F}_{B}} \\
& +\underbrace{\left(\frac{U R}{D}\right)\left(\frac{1+C_{m}}{\beta+C_{m}}\right) \tilde{\boldsymbol{u}} \cdot \nabla \tilde{\boldsymbol{u}}}_{\boldsymbol{F}_{I+A}} \\
& +\underbrace{\left(\frac{U R}{D}\right)\left(\frac{C_{L}}{\beta+C_{m}}\right)(\tilde{\boldsymbol{u}}-\tilde{\boldsymbol{v}}) \times \tilde{\boldsymbol{\omega}}}_{\boldsymbol{F}_{S}}
\end{aligned}
$$

where $\tau_{R}=R^{2} / D$ is the time taken for the particle to diffuse to the radius of the microsphere and $\tau_{B}=\left(m_{p}+m_{a}\right) /\left(6 \pi \mu_{f} r_{p}\right)$ is the Brownian relaxation time. The time step has been set 
TABLE I. Simulation parameters for numerical simulations for $r_{p}=10,50$, and $100 \mathrm{~nm}$ particles and $U=10^{-4}-6 \mathrm{~m} / \mathrm{s}$.

\begin{tabular}{lcccccc}
\hline \hline$\rho_{p}\left(\mathrm{~kg} \mathrm{~m}^{-3}\right)$ & $\mu_{f}(\mathrm{Pas})$ & $\rho_{f}\left(\mathrm{~kg} \mathrm{~m}^{-3}\right)$ & $T(\mathrm{~K})$ & $\lambda(\mathrm{m})$ & $\alpha$ & $R(\mathrm{~m})$ \\
\hline 1000 & $1.8 \times 10^{-5}$ & 1.22 & 308 & $65 \times 10^{-9}$ & $\pi / 60$ & $10 \times 10^{-6}$ \\
\hline \hline
\end{tabular}

to $\tau_{B} / 2$ to resolve the Brownian timescales. We can therefore identify five dimensionless groups: $\tau_{R} / \tau_{B}, C_{c}, \beta=\rho_{p} / \rho_{f}$, $U R / D$, and $|\boldsymbol{v}|^{\prime} / U$, where

$$
|\boldsymbol{v}|^{\prime}=\sqrt{\frac{3 k_{b} T_{f}}{m_{p}+m_{a}}} .
$$

The drag force can be further decomposed into $\boldsymbol{F}_{D}=\boldsymbol{F}_{D \boldsymbol{u}}+$ $\boldsymbol{F}_{D \boldsymbol{v}}$, which are the contributions due to the bulk flow and the particle velocities, respectively. When $U /|\boldsymbol{v}|^{\prime} \rightarrow 0$, Brownian motion is important. This nondimensionalization is different from Ref. [20], which compared Brownian and turbulent diffusion, which is not appropriate for this study.

\section{Numerical simulations}

In this work we use numerical simulations to investigate the narrow escape problem with internal circulation. The methodology is validated by numerical integration of (14) in a quiescent, unbounded domain $(\boldsymbol{u}=\mathbf{0})$ and comparing the velocity and root-mean-square (rms) displacement statistics against theoretical predictions. Results of this validation study are given in the Appendix.

The large number of variations of this problem (as will be discussed later) means that we have chosen three radii and only one size escape window $(2 \alpha=\pi / 30)$ and focused on the effect of varying the strength of the internal circulation. Due to the internal circulation resulting in a symmetrical flow field, two escape windows were used to maintain symmetry of the problem. To give this work biological context the radii of the particles were chosen to be 10,50 , and $100 \mathrm{~nm}$ within a microsphere of $R=10 \times 10^{-6} \mathrm{~m}$. The microsphere speeds studied included the range found in the human body, which is approximately $10^{-4}-1 \mathrm{~m} / \mathrm{s}$. The fluid within the microsphere was assumed to be air (see Table I). Here 200000 particles were released at the origin and were tracked for every parameter set, and averages were taken. To conform to the no-flux conditions, particles perform an elastic collision with the boundaries.

\section{RESULTS}

\section{A. Particles released from the origin}

In Fig. 2 the nondimensionalized mean escape time $\bar{T} D / R^{2}$ is plotted against varying internal strength $U /|\boldsymbol{v}|^{\prime}$ where three regimes can be identified. Regime I occurs when the internal circulation is very low (i.e., $U /|\boldsymbol{v}|^{\prime} \rightarrow 0$ ) so that the problem reduces to that of a narrow escape problem with two windows. Agreement with the asymptotic expression given in Ref. [18] is good. For Regimes II and III, the scalings for the mean escape time are $\sim\left(U /|\boldsymbol{v}|^{\prime}\right)^{-2 / 3}$ and $\sim\left(U /|\boldsymbol{v}|^{\prime}\right)^{-2}$, respectively. To gain more insight into Regimes II and III, the histograms of the escape time are plotted in Figs. 3(a)-3(c). In Regime II, for low $U /|\boldsymbol{v}|^{\prime}$ (Regime IIa), the histogram plot is similar to that in Regime I but is more concentrated at lower values of $\bar{T} D / R^{2}$ [7]. For Regime II, but higher $U /|\boldsymbol{v}|^{\prime}$ (Regime IIb), the histograms indicate a multimodal distribution. By plotting the particle trajectories [see Fig. 3(e)] it is possible to see that in this regime the particle is no longer influenced by Brownian forcing [in contrast to Regime IIb; see Fig. 3(d)] resulting in it being convected by the bulk flow and towards the escape window. However, each time it passes the escape window it will not necessarily escape, and therefore if it does not, it will be convected around the hemisphere until it does, resulting in the observed distribution. When $U /|\boldsymbol{v}|^{\prime}>1$, the particle is convected towards the stagnation point and around the edge

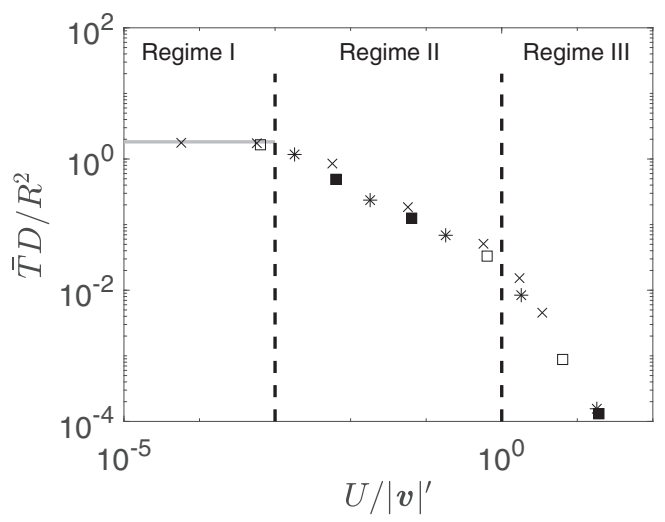

(a)

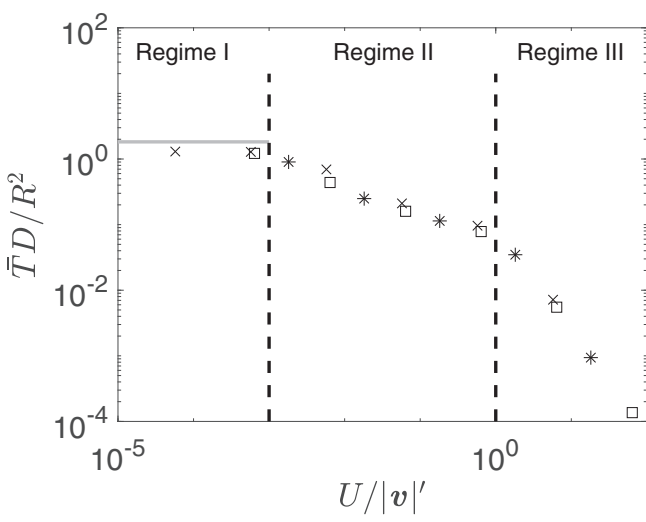

(b)

FIG. 2. Plot of variation of the nondimensional average escape time $\bar{T} D / R^{2}$ with $U /|\boldsymbol{v}|^{\prime}$ for a particle starting at (a) $\boldsymbol{x}=\{0,0\}$ and (b) $\boldsymbol{x}=$ $\{0,0.707 a\}$ [point A in Fig. 1(b)]. In Regimes II and III the scaling is $\left(U /|\boldsymbol{v}|^{\prime}\right)^{-2 / 3}$ and $\left(U /|\boldsymbol{v}|^{\prime}\right)^{-2}$, respectively. The symbols denote $10 \mathrm{~nm}$ $(\times), 50 \mathrm{~nm}(\square)$, and $100 \mathrm{~nm}(*)$ radii particles, with the histograms of the escape time for the filled squares shown in Figs. 3(a)-3(c). The gray line is the asymptotic mean escape time with two windows and no background flow [21]. 


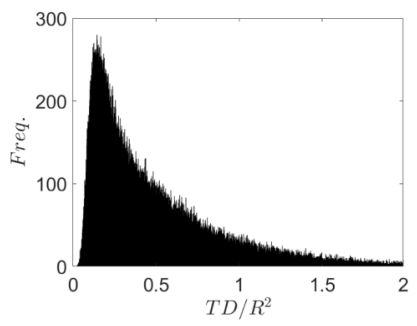

(a)

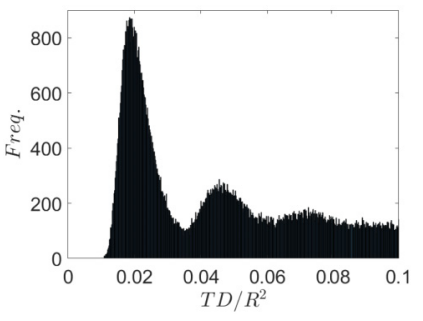

(b)

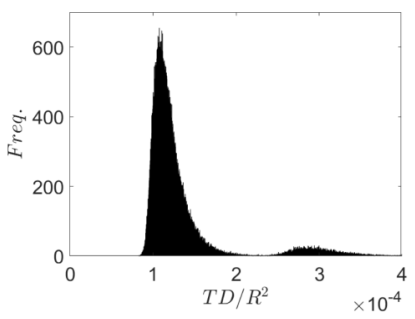

(c)

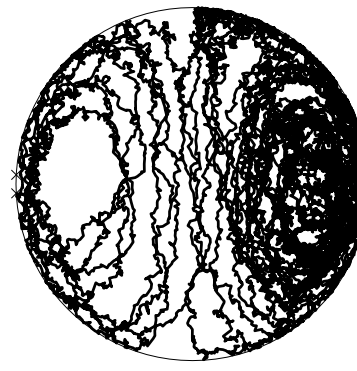

(d)

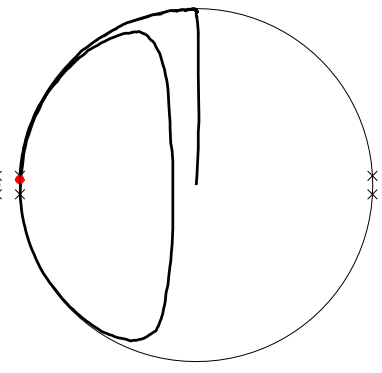

(e)

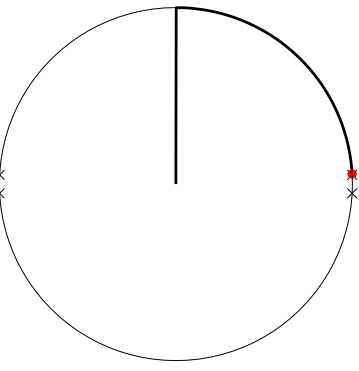

(f)

FIG. 3. Histogram of the nondimensional escape time [(a)-(c)] and typical particle trajectories [(d)-(f)] for the $50 \mathrm{~nm}$ radius particle for $U /|\boldsymbol{v}|^{\prime}=0.006$ (a, d), 0.06 (b, e), and 19 (c, f) (filled squares in Fig. 2). In panel (e) the particle has an escape time of $T D / R^{2} \approx 0.045$ showing the multimodal nature in Regime II. The red circle in panels (d)-(f) marks where the particle leaves the domain (between the two $\times$ 's, which denote the edges of the escape window).

of the hemisphere and then (with high probability) escapes immediately at the upper most point of the escape window [see Figs. 3(c) and 3(f)].

For low $U /|\boldsymbol{v}|^{\prime}, \boldsymbol{F}_{B}$ and $\boldsymbol{F}_{D v}$ are the dominant terms. In Regime II, $\boldsymbol{F}_{D u}$ is also significant, which can be seen in Fig. 3(d) where the particle is advected by the background flow but is also subjected to the random motions associated with Brownian motion. For high $U /|\boldsymbol{v}|^{\prime}$ the inertial term plays a role in removing the particle out of the domain. The lift force does not play a major role for these parameters. However, this is significant only for the largest particle (i.e., $100 \mathrm{~nm}$ radius particle). Therefore to summarize

$$
\begin{aligned}
\text { Regime I: } & U /|\boldsymbol{v}|^{\prime}<10^{-3}, \quad \boldsymbol{F}_{B} \sim \boldsymbol{F}_{D \boldsymbol{v}} \gg \boldsymbol{F}_{D \boldsymbol{u}} \sim \boldsymbol{F}_{I+A} \sim \boldsymbol{F}_{S}, \\
\text { Regime II: } & 10^{-3}<U /|\boldsymbol{v}|^{\prime}<1, \quad \boldsymbol{F}_{B} \sim \boldsymbol{F}_{D \boldsymbol{v}} \sim \boldsymbol{F}_{D \boldsymbol{u}} \gg \boldsymbol{F}_{I+A} \sim \boldsymbol{F}_{S}, \\
\text { Regime III: } & U /|\boldsymbol{v}|^{\prime}>1, \quad \boldsymbol{F}_{B} \sim \boldsymbol{F}_{D \boldsymbol{v}} \sim \boldsymbol{F}_{D \boldsymbol{u}} \sim \boldsymbol{F}_{I+A} \gg \boldsymbol{F}_{S} .
\end{aligned}
$$

\section{B. Effect of particle starting position}

Additional numerical simulations were carried out to investigate the effect of particle starting position on the average escape time. Particles were released at locations between the origin and point A in Fig. 1(b). As the results were quite similar to those released at the center of the microsphere, only the results from particles released at point $\mathrm{A}$ are shown in Fig. 2(b). For $U /|\boldsymbol{v}|^{\prime} \rightarrow 0$, there is a decrease in the mean escape time as the particle is moved towards the escape window. For example, a $10 \mathrm{~nm}$ particle released at the origin takes (on average) $35 \%$ longer than a particle released at point A. However, for $U /|\boldsymbol{v}|^{\prime}>1$, the mean escape time is greater for particles released from the center of the vortical region (i.e., point A). This is because there is zero background flow at this point and the particle first needs to diffuse due to Brownian motion to get advected towards the escape window.

TABLE II. Velocity statistics for the numerical simulations compared to the theoretical values given in (A1).

\begin{tabular}{lcccccc}
\hline \hline \multirow{2}{*}{ Radius $(\mathrm{nm})$} & \multicolumn{7}{c}{ Velocity $\left(\mathrm{ms}^{-1}\right)$} \\
\cline { 2 - 7 } & $v_{1}^{\prime}$ & $v_{2}^{\prime}$ & $w_{3}^{\prime}$ & $\sqrt{k_{b} T_{f} /\left(m_{p}+m_{a}\right)}$ & $|\boldsymbol{v}|^{\prime}$ & $\sqrt{3 k_{b} T_{f} /\left(m_{p}+m_{a}\right)}$ \\
\hline 10 & 1.02 & 1.02 & 1.02 & 1.01 & 1.76 & 1.74 \\
50 & 0.09 & 0.09 & 0.09 & 0.09 & 0.16 & 0.16 \\
100 & 0.032 & 0.032 & 0.032 & 0.032 & 0.055 \\
\hline \hline
\end{tabular}




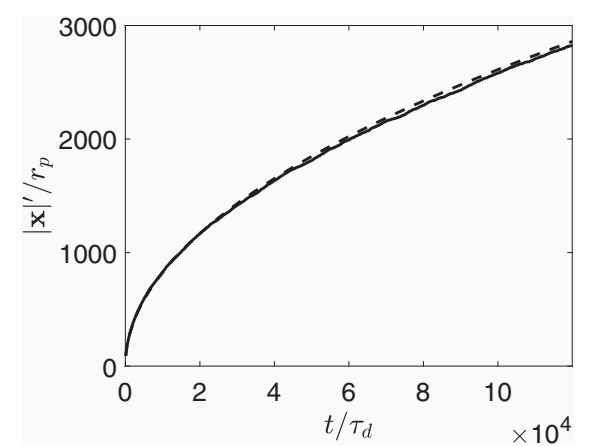

(a)

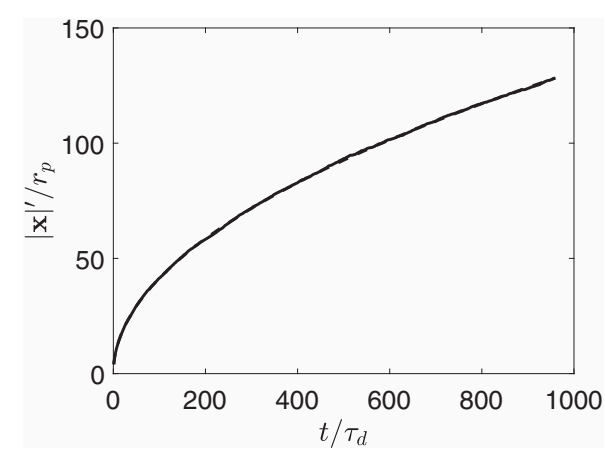

(b)

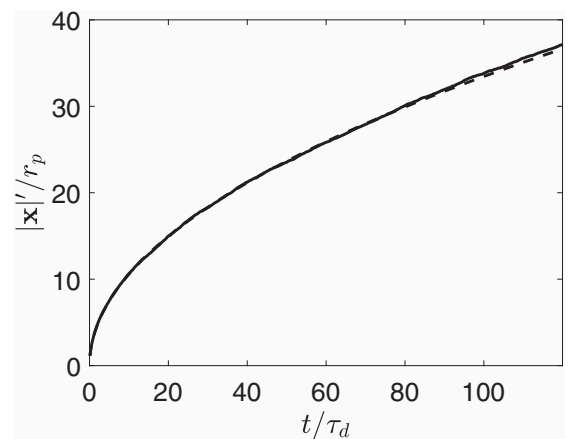

(c)

FIG. 4. Root-mean-square displacement of a (a) $10 \mathrm{~nm}$, (b) $50 \mathrm{~nm}$, and (c) $100 \mathrm{~nm}$ particle with time, where $\tau_{d}=6 \pi \mu r_{p}^{3} /\left(k_{b} T_{f}\right)$. The dashed line is the theoretical prediction (A2).

\section{DISCUSSION AND CONCLUSIONS}

This work has studied the narrow escape problem for a Brownian particle with a background flow, which is modeled with the internal flow of a Hill's vortex. Three different regimes are identified. For low $U /|\boldsymbol{v}|^{\prime}$, the particle undergoes Brownian motion, and good agreement is found with the asymptotic narrow escape relationship. As the internal circulation is increased, the bulk flow has a significant effect on the mean escape time, scaling first as $\left(U /|\boldsymbol{v}|^{\prime}\right)^{-2 / 3}$ and then $\left(U /|\boldsymbol{v}|^{\prime}\right)^{-2}$. A general description for the particle motion was given, and although not all forces were significant (i.e., the shear-induced lift force), it serves as a general framework for future studies. Due to the computational expense of the numerical simulations, these problems could benefit from future investigations into analytical solutions.

There are many more variations of the problem which can be explored including three dimensions and different number and size of the escape window. Therefore, this work should be seen as a preliminary study, and further work could include the following. First, the circulation within the microsphere was considered to be inviscid. This is a relatively good approximation for a gas bubble (as studied here) except for the thin boundary layers adjacent to the surface [22]. However, for liquid microspheres, this assumption is less valid as the viscosity of the liquid needs to be taken into account. These corrections to the velocity field could be made by using the theory in Refs. [23,24]. Additional unsteady effects of the microsphere decelerating or accelerating through a straining flow will result in either spin up or spin down of the internal circulation, which could be studied [25]. The motion of Brownian particles is significantly affected by the presence of boundaries, whether no-slip or slip boundary conditions are applied, and this could be considered in the future [26].

Drug delivery using bubbles and microgels which respond to mechanical stimuli, including compression, tension, and shear, are seen as a potential method for increasing therapeutic efficacy [27,28]. Therefore one potential application of this work is drug delivery from a microsphere; as the microsphere moves through the body, the strength of the internal circulation will be dependent on the speed of the microsphere. As was shown, the strength of the internal circulation has a profound influence on the mean escape time. Therefore, this hydrodynamic effect could be exploited in future drug delivery methods.

\section{ACKNOWLEDGMENTS}

The author acknowledges the use of the UCL Myriad High Performance Computing Facility (Myriad@UCL), and associated support services, in the completion of this work.

\section{APPENDIX}

This validation study focused on the particle parameters given in Table I. Here 2000 particles were released and tracked in time. The diagnostics are the velocity statistics and the rms displacement of the particles with time. The velocity statistics can be deduced from the equipartition theorem

$$
v_{1}^{\prime}=v_{2}^{\prime}=v_{3}^{\prime}=\sqrt{\frac{k_{b} T_{f}}{m_{p}+m_{a}}} .
$$

The rms displacement is given by

$$
|x|^{\prime}=\sqrt{6 D t} .
$$

Table II and Fig. 4 show the comparison of the velocity statistics and rms displacement between the numerical simulations and the theoretical values, respectively. Agreement is good in all cases.
[1] J. W. S. Rayleigh, The Theory of Sound, 2nd ed. (Dover, New York, 1945), Vol. 2.

[2] Z. Schuss, A. Singer, and D. Holcman, The narrow escape problem for diffusion in cellular microdomains, Proc. Natl. Acad. Sci. USA 104, 16098 (2007).
[3] J. Reingruber and D. Holcman, Diffusion in narrow domains and application to phototransduction, Phys. Rev. E 79, 030904(R) (2009).

[4] A. F. Cheviakov, A. S. Reimer, and M. J. Ward, Mathematical modeling and numerical computation 
of narrow escape problems, Phys. Rev. E 85, 021131 (2012).

[5] F. Rojo, S. W. Horacio, and C. E. Budde, The narrow escape problem: The imperfect trapping case, Phys. Rev. E 86, 031105 (2012).

[6] J.-F. Rupprecht, O. Benichou, D. S. Grebenkov, and R. Voituriez, Kinetics of active surface-mediated diffusion in spherically symmetric domains, J. Stat. Phys. 147, 891 (2012).

[7] D. Holcman and Z. Schuss, The narrow escape problem, SIAM Rev. 56, 213 (2014).

[8] C. Caginalp, Analytical and numerical results on escape of Brownian particles, Bachelor's thesis, University of Pittsburgh, 2011.

[9] T. Lagache and D. Holcman, Extended narrow escape with many windows for analyzing viral entry into the cell nucleus, J. Stat. Phys. 166, 244 (2017).

[10] H. Wang, J. Duan, X. Geng, and Y. Chao, Mean escape time for randomly switching narrow gates in a cellular flow, arXiv:1812.00800 (2018).

[11] S. M. Ghiaasiaan and G. F. Yao, A theoretical model for deposition of aerosols in rising spherical bubbles due to diffusion, convection and inertia, Aero. Sci. Tech. 26, 141 (1997).

[12] T. S. Laker and S. M. Ghiaasiaan, Monte-Carlo simulation of aerosol transport in rising spherical bubbles with internal circulation, Aero. Sci. 35, 473 (2004).

[13] I. Eames and M. A. Gilbertson, The settling and dispersion of small dense particles by spherical vortices, J. Fluid Mech. 498, 183 (2004).

[14] B. I. Shraiman, Diffusive transport in a Rayleigh-Benard convection cell, Phys. Rev. A 36, 261 (1987).

[15] M. J. M. Hill, On a spherical vortex, Philos. Trans. R. Soc. London A 185, 213 (1894).

[16] X. Bian, C. Kim, and G. E. Karniadakis, 111 years of Brownian motion, Soft Matter 12, 6331 (2016).
[17] H. Ounis, G. Ahmadi, and J. B. McLaughlin, Brownian diffusion of submicrometer particles in the viscous sublayer, J. Colloid Inter. Sci. 143, 266 (1990).

[18] A. Li and G. Ahmadi, Dispersion and deposition of spherical particles from point sources in a turbulent channel flow, Aero. Sci. Tech. 16, 209 (1992).

[19] T. R. Auton, J. C. R. Hunt, and M. Prud'homme, The force exerted on a body in inviscid unsteady non-uniform rotational flow, J. Fluid Mech. 197, 241 (1988).

[20] H. Ounis and G. Ahmadi, A comparison of Brownian and turbulent diffusion, Aero. Sci. Tech. 13, 47 (1990).

[21] S. Pillay, M. J. Ward, A. Pierce, and T. Kolokolnikov, An asymptotic analysis of the mean first passage time for narrow escape problems, SIAM J. Multiscale Mod. Sim. 8, 803 (2010).

[22] D. W. Moore, The boundary layer on a spherical gas bubble, J. Fluid Mech. 16, 161 (1963).

[23] J. Hadamard, Mouvement permanent lent dune sphere liquide et visqueuse dans un liquide visqueux, CR Acad. Sci. 152, 1735 (1911).

[24] W. Rybzynski, Ueber die fortschreitende Bewegung einer fluessigen Kugel in einem zehen Medium, Bull. Acad. Sci. Cracovie A, 40 (1911).

[25] F. A. Morrison and M. B. Stewart, Small bubble motion in an accelerating liquid, J. Appl. Mech. 43, 399 (1992).

[26] A. Mo and T. Raizen, Highly resolved Brownian motion in space and in time, Ann. Rev. Fluid Mech. 51, 403 (2019).

[27] M. W. Chang, E. Stride, and M. Edirisinghe, Stimulusresponsive liquids for encapsulation storage and controlled release of drugs from nano-shell capsules, J. Roy. Soc. Int. 8, 451 (2010).

[28] J. Wang, J. A. Kaplan, Y. L. Colson, and M. W. Grinstaff, Mechanoresponsive materials for drug delivery: Harnessing forces for controlled release, Adv. Drug Deliv. Rev. 108, 68 (2017). 